\title{
Vorwort des Autors
}

Der vorliegende Band ist das Ergebnis eines längeren Forschungsprozesses, der von einem initialen Scheitern geprägt war. Ursprünglich sollte die Lernwelt Wissenschaftliche Bibliothek auf der Grundlage einer Metaanalyse vorhandener Studien zu lernraumspezifischen Erwartungen von Bibliotheksnutzerinnen und Bibliotheksnutzern untersucht werden. Dieses Konzept ging von der Erwartung aus, dass erstens im deutschsprachigen Raum genug aktuelle Studien vorhanden sein würden und diese zweitens qualitativ und in der Aussagekraft ihrer Ergebnisse eine ausreichende Basis für eine solche Metaanalyse bieten würden. Attraktiv daran war auch die Tatsache, dass diese Methode in der bibliothekswissenschaftlichen Annäherung an die Bibliothek als Raum bisher nicht genutzt wurde. Tatsächlich wurde die erste Erwartung erfüllt, ein Aufruf über einschlägige Mailinglisten erfuhr sehr viel positive Resonanz, so dass schnell die Daten von mehr als 15 Befragungen aus den letzten fünf Jahren vor dem Aufruf zusammenkamen. Leider stellte sich schnell heraus, dass - teils aus methodischen Gründen, teils aufgrund inhaltlicher Schwächen - eine Vergleichbarkeit der Ergebnisse nicht gegeben war. Auf der Grundlage der vorliegenden Daten konnten keine substanziellen Aussagen zur Lernwelt Wissenschaftliche Bibliothek gewonnen werden. Es zeigte sich, dass nach wie vor keine ausreichende theoretische Grundlage gegeben war, die in den Fragestellungen $\mathrm{zu}$ wirklich neuen Ergebnissen hätte führen können und dass viele Befragungen im Forschungsdesign und in der Auswertung methodisch unbefriedigend waren. Dies führte zu einer grundlegenden Neukonzeption des gesamten Bandes.

Schnell wurde deutlich, dass ein Band zur Lernwelt Wissenschaftliche Bibliothek innerhalb der Reihe Lernwelten das Thema zunächst theoretisch durchdringen müsste, um für die zukünftige Auseinandersetzung - sowohl in der Theorie als auch in der bibliothekarischen und hochschulplanerischen Praxis eine ausreichende Basis zu schaffen. Ganz im Sinne der Zielsetzung der Reihe sollte eine interdisziplinäre und vor allem auch internationale Perspektive eröffnet werden. Der hierfür notwendige Ansatz erforderte einen langen Atem und das vorliegende Ergebnis ist wesentlich auch der Geduld des Reihenherausgebers zu verdanken, da mehrere Verschiebungen notwendig wurden.

Die Fertigstellung des Bandes fiel schließlich in die Zeit der COVID-19-Pandemie, in deren Verlauf zunehmend Fragen zum zukünftigen Verhältnis digitaler und physischer Umgebungen nicht nur in der Lernwelt Wissenschaftliche Bibliothek gestellt wurden. Aussagen über die langfristigen Folgen der Pandemie sind gegenwärtig nicht seriös zu treffen. Die in diesem Band behandelten Fra- 
X - Vorwort des Autors

gen haben aber im Zuge einer fortschreitenden Digitalisierung der Lehre und dreier Semester mit wenig oder keinen Präsenzveranstaltungen und einem reduzierten Angebot physischer Lernräume in den Hochschulen eine neue Relevanz bekommen.

Im Juni 2021

Olaf Eigenbrodt 